\title{
Safety and feasibility of laparoscopic sleeve gastrectomy with loop duodenojejunal bypass for obesity
}

\author{
Amar Vennapusa', Ramakanth Bhargav Panchangam², Charita Kesara ${ }^{1}$, Nazneen Mallick \\ 'Department of Bariatric and Metabolic Surgery, Dr. Amar Bariatric \& Metabolic Center, Hyderabad, Telangana, India \\ ${ }^{2}$ Department of Endocrine and Metabolic Surgery, Endocare Hospital, Vijayawada, India
}

Purpose: Laparoscopic sleeve gastrectomy (SG) with loop duodenojejunal bypass (LDJB) is a combination of SG and one anastomosis gastric bypass surgeries. This study was intended to evaluate the safety and feasibility of SG-LDJB surgery.

Methods: This was a retrospective study analyzing SG-LDJB surgery using 250-cm length biliopancreatic limbs that was performed between May 2013 and December 2017 in 113 Indians with obesity. All demographic, clinical, operative, and follow-up data were analyzed for weight loss and diabetes remission efficacy. Weight-loss success was defined as percentage of total weight loss (\%TWL) $\geq 25 \%$. Diabetes remission was defined as hemoglobin Alc $<6 \%$ without the need for antidiabetic medications. Safety and feasibility were analyzed in terms of intraoperative and postoperative complications and serum albumin, iron, and calcium levels.

Results: The \%TWL was $35.0 \%$ and $31.0 \%$ at the 1-year and 3-year follow-ups, respectively. The weight-loss success rate was $91.3 \%$ and $78.1 \%$ at the 1-year and 3-year follow-ups, respectively. Sixty-six out of 113 patients had type 2 diabetes. Diabetes remission was $80.0 \%$ and $75.4 \%$ at the 1-year and 3-year follow-ups, respectively. Major perioperative complications occurred in $0.9 \%$ of the patients. The 30 -day mortality rate was zero. None of the patients had serum albumin levels of $<3 \mathrm{~g} / \mathrm{dL}$ at the 1-year and 3-year follow-ups or symptoms of hypoproteinemia.

Conclusion: SG-LDJB surgery using a $250-\mathrm{cm}$ biliopancreatic limb is a safe and effective procedure to treat obesity. It is technically complex but has the advantages of endoscopic access to the stomach and negligible postoperative complications or hypoproteinemia.
Received July 30, 2020

Revised November 10, 2020

Accepted December 1,2020

Corresponding author

Amar Vennapusa

Department of Bariatric and

Metabolic Surgery, Dr. Amar Bariatric

\& Metabolic Center, Plot No - 99,

Ground Floor, Next to Chiranjeevi

Blood Bank, Opposite to Metro

Pillar C1588, Road No - 1, Jubilee

Hills Check Post Road, Jubilee Hills,

Hyderabad, Telangana 500033, India

Tel: +91-96766-75646

E-mail:drVamar@gmail.com

ORCID:

https://orcid.org/0000-0001-7338-1922

Keywords: Bariatric surgery, Diabetes mellitus, Metabolic surgery, Obesity, Weight loss

This is an Open Access article distributed under the terms of the Creative Commons Attribution Non-Commercial License (http:// creativecommons.org/licenses/by-nc/4.0/) which permits unrestricted non-commercial use, distribution, and reproduction in any medium, provided the original work is properly cited.
Copyright (C) 2021 The Journal of Minimally Invasive Surgery. All rights reserved.

\section{INTRODUCTION}

Obesity is one of the most prevalent lifestyle diseases and has currently become a global pandemic [1]. Furthermore, it is one of the most frequent causes of type 2 diabetes. Attempts at managing severe obesity using methods such as diet and exercise have been found to be unsuccessful [2]. However, surgery is an effective method to manage both severe obesity and its associated comorbidities. There are several bariatric surgeries available, which suggests that no single procedure is suitable for all patients suffering from obesity [3].

Laparoscopic surgical options include sleeve gastrectomy (SG), Roux-en-Y gastric bypass (RYGB), one anastomosis gastric bypass (OAGB), and the most effective, biliopancreatic diversion (BPD) with duodenal switch (DS). One of the standard surgeries, RYGB, has several short- and long-term complications such as loss of 
access to the gastric remnant, dumping syndrome, marginal ulcers, internal hernias, and vitamin deficiencies [4]. Postoperative complication rates of RYGB and SG are 3.02\% and 2.12\%, respectively. The mortality rate was found to be between $0 \%$ and $1.5 \%$ in obese patients and higher in obese patients with comorbidities [5]. Other postoperative complications associated with bariatric surgeries include internal bleeding (2\%), leakage, and deep vein thrombosis. Long-term complications are related to nutritional deficiencies that require long-term vitamin and mineral supplementation [2].

Laparoscopic SG with loop duodenojejunal bypass (LDJB) surgery is a combination of SG and OAGB surgeries (Fig. 1). This surgery is, to some extent, aimed at addressing the limitations of standard bariatric surgeries such as SG and RYGB. It is similar to laparoscopic single anastomosis duodenoileal bypass with sleeve (SADI-S), but with a more proximal anastomosis. In SG-LDJB surgery, the jejunum, instead of the ileum, is anastomosed to the divided first part of the duodenum in a loop fashion, 200 to 250 $\mathrm{cm}$ distal to the duodenojejunal flexure. Pyloric preservation in SG-LDJB surgery reduces the risk of marginal ulcers and prevents bile reflux and dumping syndrome [4]. A reduction of marginal ulcer risk from 3\% in RYGB to $0.3 \%$ in BPD-DS, and the absence of dumping syndrome in the later, is attributed to preservation of the pylorus [6]. The reduced number of anastomoses and mesenteric defects in SG-LDJB surgery reduces the incidence of anastomotic leaks and internal hernias [4]. However, available literature regarding SG-LDJB surgeries is limited. Therefore, this study was initiated to evaluate the safety and feasibility of SGLDJB surgery using a $250-\mathrm{cm}$ biliopancreatic limb in Indian patients.

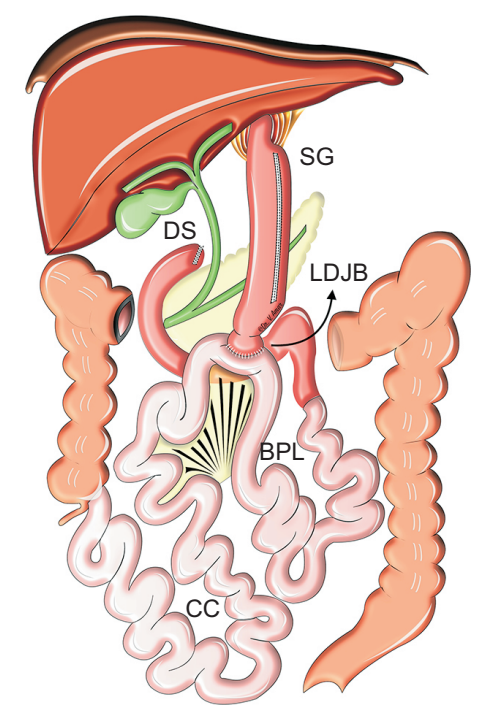

Fig. 1. Pictorial diagram of sleeve gastrectomy (SG) with loop duodenojejunal bypass (LDJB). DS, duodenal stump; BPL, biliopancreatic limb; CC, common channel.

\section{MATERIALS AND METHODS}

This was a retrospective study conducted with 113 Indian patients between May 2013 and December 2017. Patients who underwent SG-LDJB surgery with a 250-cm biliopancreatic limb for obesity were included in the study. Those who underwent SGLDJB surgery with a 200-cm biliopancreatic limb were excluded.

In our SG-LDJB surgical technique, a total of four ports were used. The greater curvature of the stomach was devascularized up to the gastroesophageal fat pad using a vessel sealer. Dissection was continued $5 \mathrm{~cm}$ beyond the pylorus along the inferior margin of the duodenum. The first part of the duodenum (D1) was mobilized all around until the gastroduodenal artery was identified. The right gastric artery was identified and divided at its origin. Blood supply to D1 from the lesser omental vascular arcade was preserved. As D1 was completely mobilized by dividing the right gastric artery, the only blood supply left to supply the gastric sleeve and D1 was the left gastric artery through the lesser omental vascular arcade. D1 was not divided immediately after completion of duodenal mobilization (Fig. 2). The gastric

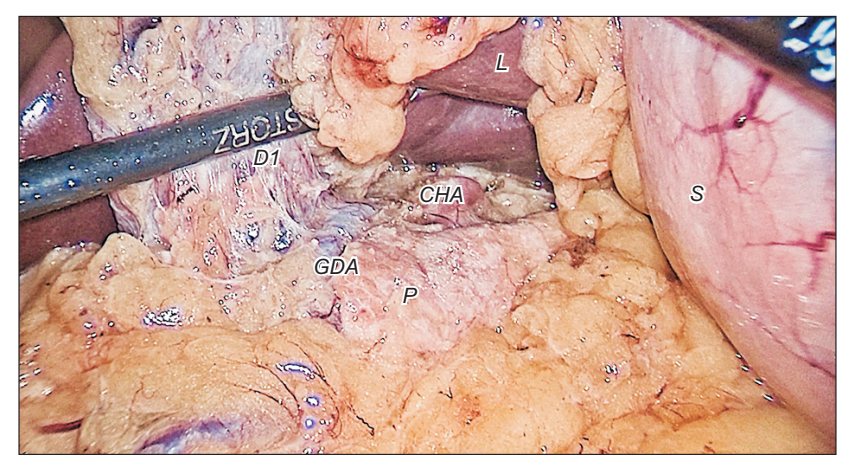

Fig. 2. Intraoperative photograph of completed duodenal dissection. L, liver; D1, first part of duodenum; CHA, common hepatic artery; S, stomach; GDA, gastroduodenal artery; P, pancreas.

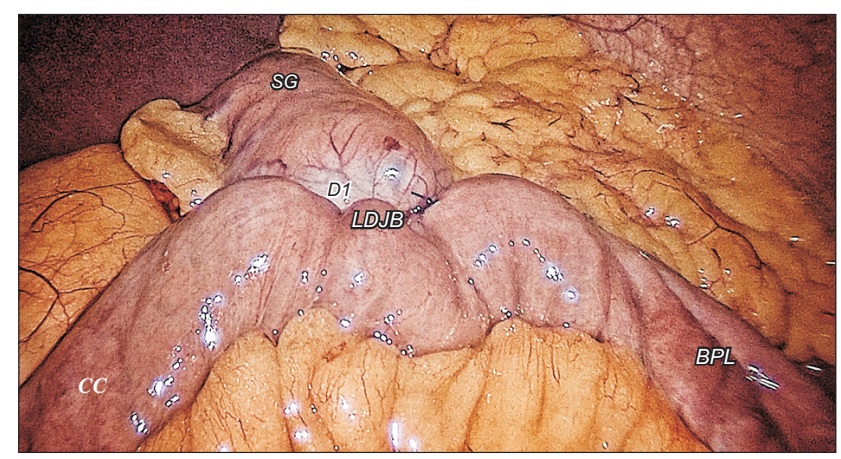

Fig. 3. Intraoperative photograph of loop duodenojejunal bypass (LDJB). SG, sleeve gastrectomy; D1, first part of duodenum; BPL, biliopancreatic limb; CC, common channel. 
sleeve was fashioned around a 12.7-mm gastric calibration tube starting $4 \mathrm{~cm}$ proximal to the pylorus. After completion of the gastric sleeve formation, D1 was transected 2 to $4 \mathrm{~cm}$ distal to the pylorus using staples. The division of the right gastric artery facilitated free mobility of $\mathrm{D} 1$ and the pyloric antrum. The jejunum, at the $250-\mathrm{cm}$ mark from the duodenojejunal flexure, was anastomosed to D1 in an antecolic loop fashion using 3-0 absorbable sutures in four layers (Fig. 3). The remaining bowel length was measured to ensure that the common channel length was a minimum of $400 \mathrm{~cm}$.

Patients were ambulated on the first postoperative day. A leak check was performed via a contrast X-ray of the stomach, an ultrasound scan of the abdomen, and a complete blood picture 1 week and 1 month following surgery. Patients were analyzed on the basis of weight, body mass index (BMI), percentage of total weight loss (\%TWL), percentage of excess weight loss (\%EWL) using a BMI reference point of $25 \mathrm{~kg} / \mathrm{m}^{2}$, and hemoglobin Alc (HbAlc) levels at 1-year and 3-year follow-ups. Weight-loss success was defined as $\% \mathrm{TWL} \geq 25 \%$.

Complete diabetes remission was defined as $\mathrm{HbAlc}<6 \%$ with complete cessation of insulin or oral hypoglycemic agents $[7,8]$. Partial diabetes remission was defined as HbAlc $\geq 6 \%$ and $<6.5 \%$ in the absence of antidiabetic medications. Diabetes improvement was defined as a statistically significant reduction in HbAlc that did not meet the criteria for complete or partial remission or a decrease in the requirements for antidiabetic medications. $A B C D$ scores were measured, and complete diabetes remission was analyzed with respect to this score using logistic regression analysis. Blood pressure that was within normal limits without the need for medications was considered as a remission of hypertension following surgery. Serum lipid levels that were within normal limits without the need for lipid-lowering medications was considered as a remission of hyperlipidemia following surgery. In those individuals with documented osteoarthritis, improvement in joint pain was considered to be a favorable response to surgery. In patients suffering from obstructive sleep apnea, the disappearance or improvement in symptoms such as snoring, frequent awakening, nocturia, and daytime somnolence was considered a favorable response to surgery. These responses were documented at the 1-year and 3-year follow-ups.

Serum albumin levels were measured and analyzed at the 1-year and 3-year follow-ups. Hypoalbuminemia was defined as serum albumin levels $<3.5 \mathrm{~g} / \mathrm{dL}$. Hypoalbuminemia was considered mild when serum albumin levels were $\geq 3 \mathrm{~g} / \mathrm{dL}$ and $<3.5 \mathrm{~g} / \mathrm{dL}$ and moderate when the levels were $\geq 2.5 \mathrm{~g} / \mathrm{dL}$ and $<3 \mathrm{~g} / \mathrm{dL}$. Severe hypoalbuminemia was defined as serum albumin levels $<2.5 \mathrm{~g} /$ dL. Serum iron, calcium, and hemoglobin levels were also measured and analyzed at the 1-year and 3-year follow-ups. Serum iron levels $<50 \mu \mathrm{g} / \mathrm{dL}$ in females and $<65 \mu \mathrm{g} / \mathrm{dL}$ in males were considered low. Calcium deficiency was considered when serum calcium levels were $<8.8 \mathrm{~g} / \mathrm{dL}$. Anemia was defined as hemoglobin levels $<12 \mathrm{~g} / \mathrm{dL}$ in females and $<13 \mathrm{~g} / \mathrm{dL}$ in males. IBM SPSS version 23 (IBM Corp., Armonk, NY, USA) was used to perform the statistical analysis. The $p$ values of $<0.05$ were considered statistically significant.

\section{RESULTS}

Between May 2013 and December 2017, SG-LDJB surgery was performed on 126 patients with obesity. Of these, 113 had biliopancreatic limb lengths fixed at $250 \mathrm{~cm}$, and their data were analyzed. Intraoperatively, 16 patients developed mild duodenal discoloration, which disappeared by the end of surgery. None of the patients developed D1 necrosis. Six patients (5.3\%) had umbilical hernias of $<5 \mathrm{~cm}$, which were repaired with interrupted, nonabsorbable No. 1-0 prolene sutures. Mesh repair was deferred given concomitant duodenojejunal anastomosis. Eleven patients (9.7\%) underwent concomitant adhesiolysis, and six patients (5.3\%) underwent concomitant laparoscopic cholecystectomies. Intraoperative complications occurred in three patients (2.7\%). In one patient, the common hepatic duct was injured during the cholecystectomy. This was sutured with interrupted 4-0 polydioxanone sutures. An accidental deep pancreatic head injury occurred in one patient and a full thickness injury of the $\mathrm{D} 1$ in another. The duodenal injury was included in the opening created for duodenojejunal anastomosis. These three patients recovered well without postoperative complications.

The mean hospital stay was 3 days (range, 2-7 days). Major postoperative complications occurred in one patient $(0.9 \%)$. This patient developed extensive portal vein thrombosis on the tenth postoperative day, which occurred despite keeping the patient on prophylactic anticoagulation with factor Xa inhibitors. The patient was treated conservatively with long-term oral factor Xa inhibitors. There was no leakage or deep vein thrombosis in any of the patients and the 30-day mortality rate was zero.

There were no major intraoperative or postoperative intraabdominal bleeding complications. Two patients were found to have small hematomas adjacent to the duodenal stump, at the site of gastroduodenal artery, 2 weeks postsurgery. These were most likely due to postoperative bleeding from the divided branches of the gastroduodenal artery. The hematomas resolved spontaneously. One patient developed ecchymosis over the right flank, as a complication of postoperative anticoagulation. This resolved after anticoagulants were stopped.

Two patients developed pneumonia, which resolved after the administration of broad-spectrum antibiotics. Postoperative milestones of 1-year and 3-year follow-ups were achieved in 113 and 91 patients, respectively. Of these, $92(81.4 \%)$ and $73(80.2 \%)$ were available at the 1-year and 3-year follow-ups, respectively. Twenty-one (18.6\%) and 18 (19.8\%) patients were lost to follow-up 
Table 1. Weight parameters

\begin{tabular}{|c|c|c|c|c|}
\hline Parameter & Time & Number & Value & $p$ value $^{\mathrm{a})}$ \\
\hline \multirow[t]{3}{*}{ Weight (kg) } & Preoperative (A) & 113 & $117.35 \pm 15.62$ & \\
\hline & 1 yr (B) & 92 & $76.06 \pm 11.4$ & $<0.001(\mathrm{~A}-\mathrm{B})$ \\
\hline & 3 yr (C) & 73 & $79.52 \pm 12.54$ & $<0.001(A-C)$ \\
\hline \multirow[t]{3}{*}{ Body mass index $\left(\mathrm{kg} / \mathrm{m}^{2}\right)$} & Preoperative (A) & 113 & $41.21 \pm 5.73$ & \\
\hline & $1 \mathrm{yr}(\mathrm{B})$ & 92 & $26.92 \pm 3.85$ & $<0.001(A-B)$ \\
\hline & 3 yr (C) & 73 & $28.45 \pm 4.37$ & $<0.001(\mathrm{~A}-\mathrm{C})$ \\
\hline \multirow[t]{2}{*}{$\%$ TWL } & $1 \mathrm{yr}(\mathrm{B})$ & 92 & $34.96 \pm 5.99$ & \\
\hline & 3 yr (C) & 73 & $31.02 \pm 7.52$ & \\
\hline \multirow[t]{2}{*}{ \%EWL } & $1 \mathrm{yr}(\mathrm{B})$ & 92 & $95.04 \pm 25.82$ & \\
\hline & 3 yr (C) & 73 & $84.5 \pm 25.59$ & \\
\hline \multirow{2}{*}{$\begin{array}{c}\text { Weight loss success rate, } \\
\% \text { TWL } \geq 25 \%:<25 \%\end{array}$} & $1 \mathrm{yr}$ & 92 & $84: 8(91.3: 8.7)$ & \\
\hline & $3 \mathrm{yr}$ & 73 & $57: 16(78.1: 21.9)$ & \\
\hline
\end{tabular}

Values are presented as mean \pm standard deviation or number (\%).

$\%$ TWL, percentage of total weight loss; \%EWL, percentage of excess weight loss.

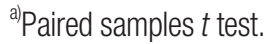

Table 2. Analysis of HbA1c and diabetes response

\begin{tabular}{clccc} 
Parameter & \multicolumn{1}{c}{ Time } & Number & Value & $p$ value \\
\hline HbA1c $(\%)$ & Preoperative (A) & 66 & $7.79 \pm 1.48$ & \\
& $1 \mathrm{yr}(\mathrm{B})$ & 65 & $5.56 \pm 0.67$ & $<0.001(\mathrm{~A}-\mathrm{B})$ \\
Response, CR:PR:DI & $3 \mathrm{yr}(\mathrm{C})$ & 61 & $5.58 \pm 0.55$ & $<0.001(\mathrm{~A}-\mathrm{C})$ \\
& $1 \mathrm{yr}$ & 65 & $52: 6: 7(80.0: 9.2: 10.8)$ & \\
\hline
\end{tabular}

Values are presented as mean \pm standard deviation or number (\%).

$\mathrm{HbA1c}$, hemoglobin A1c; CR, complete diabetes remission; PR, partial diabetes remission; DI, diabetes improvement.

a)Paired samples $t$ test.

at the 1-year and 3-year follow-ups, respectively.

The mean weights and BMIs at different time intervals are shown in Table 1. \%TWL and \%EWL (with a BMI reference point of $25 \mathrm{~kg} / \mathrm{m}^{2}$ ) were calculated at the 1-year and 3-year followups and are shown in Table 1. Weight loss was significant at the 1-year and 3-year follow-ups compared to the preoperative weight (Table 1). The weight-loss success rate was $91.3 \%$ and $78.1 \%$ at the 1 and 3-year follow-ups, respectively, with $\% \mathrm{TWL}$ of $\geq 25 \%$ taken as a reference point (Table 1). There was inadequate weight loss in $8.7 \%$ (8 of 92) and $21.9 \%$ (16 of 73) of patients at the 1-year and 3 -year follow-ups, respectively, with \%TWL falling below 25\%.

HbAlc level, complete diabetes remission, partial diabetes remission, and diabetes improvement at the 1-year and 3-year follow-ups are shown in Table 2. HbAlc was significantly lower at the 1-year and 3-year follow-ups compared to preoperative values. Complete diabetes remission was $80.0 \%$ and $75.4 \%$ at the 1-year and 3-year follow-ups, respectively. The remaining patients were either in partial diabetes remission or the diabetes improvement category at the 1-year and 3-year follow-ups (Table 2). None of the patients experienced diabetes deterioration. ABCD scores positively predicted complete diabetes remission (logistic regression analysis: $\mathrm{B}=0.771, p=0.002$ and $\mathrm{B}=1.023, p=0.001$ at the 1-year and 3-year follow-ups, respectively). Patients with higher $\mathrm{ABCD}$ scores had significantly better complete diabetes remission.

The incidence of various comorbidities and patient responses to surgery at the 1-year and 3-year follow-ups are shown in Table 3. Three patients developed cancer during the postoperative follow-up. One had carcinoma of the head of the pancreas, one had pyloric antral carcinoma, and the other had breast cancer 2 years 
Table 3. Response of comorbid conditions

\begin{tabular}{lccc|}
\hline \multirow{2}{*}{ Comorbid condition } & Patient $(\mathrm{n}=113)$ & \multicolumn{2}{c|}{ Response at follow-up } \\
\cline { 3 - 4 } & & $1 \mathrm{yr}$ & $3 \mathrm{yr}$ \\
\hline Hypertension & $45(39.8)$ & $33 / 42(78.6)$ & $31 / 39(79.5)$ \\
Hyperlipidemia & $30(26.5)$ & $18 / 27(66.7)$ & $16 / 24(66.7)$ \\
\hline Osteoarthritis & $20(17.7)$ & $12 / 18(66.7)$ & $11 / 17(64.7)$ \\
\hline Obstructive sleep apnea & $28(24.8)$ & $20 / 24(83.3)$ & $15 / 19(78.9)$ \\
\hline
\end{tabular}

Values are presented as number (\%).

following surgery. All three had obesity with type 2 diabetes before surgery. These patients received surgical treatment for their respective cancers, as well as chemotherapy. The patient with pyloric antral carcinoma lost his life due to reasons unrelated to either cancer or the bariatric surgery 3 years following surgery. The remaining two patients are currently leading active lives.

None of the patients suffered from gastroesophageal reflux disease prior to surgery. However, $3.3 \%$ (3 of 92) of the patients developed symptomatic gastroesophageal reflux disease by the 1-year follow-up, which required occasional treatment with proton pump inhibitors. None of the patients developed clinical manifestations suggestive of dumping syndrome or internal hernias. Upon investigation, $33.7 \%$ and $31.5 \%$ of patients were found to have low serum iron levels and $39.1 \%$ and $15.1 \%$ of patients had asymptomatic calcium deficiencies at the 1-year and 3-year follow-ups, respectively. Anemia was noted in $29.4 \%$ and $30.1 \%$ of patients at the 1-year and 3-year follow-up intervals, respectively. These patients responded to oral supplements. Hypoalbuminemia was observed in $13.0 \%$ and $6.9 \%$ at the 1-year and 3-year follow-ups, respectively. None of the patients had serum albumin levels of $<3 \mathrm{~g} / \mathrm{dL}$ at either follow-up period. Furthermore, none of the patients developed symptoms of protein energy malnutrition. Three patients developed chronic diarrhea at the 1-year followup, which was controlled with antispasmodics.

\section{DISCUSSION}

Bariatric surgery is a highly effective method for the treatment of obesity and type 2 diabetes. SG surgery is simple and easy to perform, but weight regain and the recurrence of comorbidities is high over time [9]. RYGB surgery is the gold standard surgery to treat obesity [10]. However, it has limitations such as a prolonged learning curve, endoscopic inaccessibility for the monitoring of the remnant stomach (at-risk gastric remnant), an increased risk of calcium and iron deficiencies (despite the administration of oral supplements) due to an excluded proximal bowel, an increased risk of dumping syndrome due to the exclusion of the pylorus, and internal hernias [11-14]. OAGB surgery has recently become quite popular because of its technical simplicity and increased effectiveness. It addresses the problem of internal hernias associated with RYGB surgery; although, the other problems mentioned persist [15]. BPD-DS is the most effective surgery, both in terms of weight loss and diabetes remission, as hormonal changes are maximized when food empties directly into the distal ileum. However, malabsorption is increased as most of the bowel is bypassed [16].

Huang et al. [17] have proposed using SG-LDJB surgery to address the limitations associated with standard surgeries such as SG and RYGB. Various loop DS surgeries have been described in the literature that use different biliopancreatic limb and common channel lengths [18]. SG-LDJB surgery is a combination of SG and OAGB surgeries using a fixed biliopancreatic limb length to address the malabsorption associated with BPD-DS surgery. To simplify the surgery, we modified the SG-LDJB surgical technique by dividing the right gastric artery at its origin and teasing the lesser omentum anterior to the caudate lobe before transecting D1. This modification helped to complete the mobilization of the pyloric antrum and D1. As a result, the gastric sleeve and divided D1 were supplied only by the left gastric pedicle and anchored by the esophagus and lesser omental vascular arcade. Advantages of this modification include being technically simple, allowing for the free movement of the pyloric antrum and D1, and facilitating tension-free anastomosis with the jejunum. Moreover, there is no need to divide the omentum to facilitate the anastomosis, and none of the patients in our study required omental division. While the resultant gap behind the loop duodenojejunal anastomosis was quite large, it probably obviates the possibility of intestinal obstruction or strangulation, even if small intestinal loops enter the gap behind the anastomosis. In SG-LDJB surgery, the loop duodenojejunal anastomosis is anatomical from end-to-side. Dallegrave Marchesini [19] has proposed the division of the right gastric artery while performing loop DS surgeries, while Gebelli et al. [20] has shown that SADI-S surgery with right gastric artery ligation can be performed safely.

While this modification is simple and facilitates tension-free anastomosis, it has few disadvantages. As the left gastric artery is the only feeding vessel to the lesser omental vascular arcade supplying D1, the blood supply to D1 may be compromised if 
the lesser omental vascular arcade branches to D1 are not preserved, which would lead to ischemia and an increased risk of leak. While transient discoloration of the duodenal mucosa was observed in some of our patients (severe discoloration warrants resection of the D1), this disappeared when the anastomosis was completed. As the majority of the nerve supply to the pylorus comes through the nerve plexus around the right gastric artery, this modification could result in vagal denervation of the pylorus, compromising the function of the pyloric sphincter in some patients.

The anastomosis of the jejunum to a divided D1 in a Rouxen-Y fashion has been described by Kasama et al. [21] (SG with duodenojejunal bypass). In comparison, our loop anastomosis is simpler and with a reduced number of anastomoses and mesenteric gaps. By using loop rather than Roux-en-Y anastomosis, antecolic rather than retrocolic anastomosis, and the division of the right gastric artery with the free lying D1, this may translate into a reduced risk of internal hernias as the number of mesenteric gaps are reduced. Endoscopic surveillance of the gastric sleeve is possible after SG-LDJB surgery; hence, there is no 'at-risk' gastric remnant. The pylorus was preserved in our surgeries, which may reduce dumping syndrome to some extent, but it cannot be entirely avoided [4].

This study demonstrates that patients can achieve significant weight loss following SG-LDJB surgery, which is sustainable over a period of several years. While there was some weight regain at the 3-year follow-up, more than $75 \%$ of patients maintained a $\% \mathrm{TWL} \geq 25 \%$, indicating that SG-LDJB surgery was highly effective in terms of weight loss. Maciejewski et al. [22] showed that weight regain after RYGB was $3.4 \%$ at the 10 -year follow-up. Nor Hanipah et al. [23] found a significant drop in BMI from 30.2 \pm 5.1 to $22.9 \pm 5.6 \mathrm{~kg} / \mathrm{m}^{2}$ after 2 years in 163 patients who underwent SG-LDJB surgery. Our study also showed that a significant percentage of patients achieved and maintained diabetes remission over a period of several years. This is also in accordance with Huang et al. [4] who showed complete and partial diabetes remission in $30 \%$ and $16.7 \%$, respectively, of RYGB surgery patients and in $36.7 \%$ and $16.7 \%$, respectively, of SG-LDJB patients at a 1-year follow-up.

The digestion and absorption of nutrients primarily occur in the duodenum and proximal jejunum [24]. The duodenum, in particular, plays an important role in the absorption of minerals under states of deficiency $[25,26]$. In SG-LDJB surgery, the calcium and iron deficiency risk is theoretically low as the D1 is preserved. While some of our patients had low serum iron levels and asymptomatic calcium deficiencies, they responded well to oral supplements. This response may be related to the preservation of D1. The major complication rate in this study was $0.9 \%$, which was low. Nor Hanipah et al. [23] had a complication rate of $3.6 \%$ after SG-LDJB surgery, which required reoperation. None of the patients in this study developed symptoms suggestive of dumping syndrome or internal hernias. Internal hernias are one of the most common complications of RYGB surgery. According to one meta-analysis, the incidence of internal hernia was found to be $1 \%$ in patients who underwent RYGB surgery with antecolic Roux limbs and closure of mesenteric and Petersen defects [4].

Obesity and diabetes increase the risk for cancer [27,28], and bariatric surgery reduces this risk [29]. In this study, three patients with obesity and diabetes were found to have cancer at the 2-year follow-up. Obesity itself, coupled with other comorbidities, was thought to be responsible for these cancers, and bariatric surgery was unable to prevent the development and progression of cancers in these patients. While SG-LDJB surgery was not performed in patients with gastroesophageal reflux disease, the incidence of postoperative gastroesophageal reflux was very low in this study. The modification of dividing the right gastric artery and keeping the sleeve and D1 in the vertical position may have reduced the reflux.

There are several limitations associated with SG-LDJB surgery. It is a technically complex procedure with a steep learning curve; access to the biliary tract, moreover, is lost in SG-LDJB surgery as the second part of the duodenum is excluded. However, our results indicate that SG-LDJB is a safe surgery to treat patients suffering from obesity and diabetes. Long-term data from multiple centers is desirable to standardize and evaluate the exact efficacy of this novel surgery.

\section{NOTES}

\section{Ethical statements}

The study was conducted in compliance with the principles of the Declaration of Helsinki. The study protocol was reviewed and approved by our Institutional Review Board (IRB No. ABMC/AH/2020/005). Detailed informed consent was obtained from all study participants. All demographic, clinical, operative, and follow-up data were documented in a structured master datasheet.

\section{Authors' contributions}

\section{Conceptualization: AV}

Data curation: AV, CK, NM

Formal analysis: All authors

Investigation: $\mathrm{AV}, \mathrm{CK}$

Methodology: AV, RBP

Project administration: AV, CK

Visualization: All authors

Writing-original draft: All authors

Writing-review \& editing: All authors 
All authors read and approved the final manuscript.

\section{Conflict of interest}

All authors have no conflicts of interest to declare.

\section{ORCID}

Amar Vennapusa, https://orcid.org/0000-0001-7338-1922

Ramakanth Bhargav Panchangam, https://orcid.org/0000-0002-8465-0558

Charita Kesara, https://orcid.org/0000-0002-1282-0087

Nazneen Mallick, https://orcid.org/0000-0002-6615-6265

\section{REFERENCES}

1. Popkin BM, Adair LS, Ng SW. Global nutrition transition and the pandemic of obesity in developing countries. Nutr Rev 2012;70:3-21.

2. Szczuko M, Komorniak N, Hoffmann M, et al. Body weight reduction and biochemical parameters of the patients after RYGB and SG bariatric procedures in 12-month observation. Obes Surg 2017;27:940947.

3. Deylgat B, D'Hondt M, Pottel H, Vansteenkiste F, Van Rooy F, Devriendt D. Indications, safety, and feasibility of conversion of failed bariatric surgery to Roux-en-Y gastric bypass: a retrospective comparative study with primary laparoscopic Roux-en-Y gastric bypass. Surg Endosc 2012;26:1997-2002.

4. Huang CK, Tai CM, Chang PC, Malapan K, Tsai CC, Yolsuriyanwong K. Loop duodenojejunal bypass with sleeve gastrectomy: comparative study with Roux-en-Y gastric bypass in type 2 diabetic patients with a BMI $<35 \mathrm{~kg} / \mathrm{m}(2)$, first year results. Obes Surg 2016;26: 2291-2301.

5. Moulla Y, Lyros O, Blüher M, Simon P, Dietrich A. Feasibility and safety of bariatric surgery in high-risk patients: a single-center experience. J Obes 2018;2018:7498258.

6. Hess DS, Hess DW, Oakley RS. The biliopancreatic diversion with the duodenal switch: results beyond 10 years. Obes Surg 2005;15:408416.

7. Cummings DE, Arterburn DE, Westbrook EO, et al. Gastric bypass surgery vs intensive lifestyle and medical intervention for type 2 diabetes: the CROSSROADS randomised controlled trial. Diabetologia 2016;59:945-953.

8. Vennapusa A, Panchangam BR, Madivada MS. A feasibility study of novel "laparoscopic sleeve gastrectomy with loop gastroileal bypass" for obesity: an Indian experience. Int Surg 2017;102:504-513.

9. Clapp B, Wynn M, Martyn C, Foster C, O’Dell M, Tyroch A. Long term (7 or more years) outcomes of the sleeve gastrectomy: a metaanalysis. Surg Obes Relat Dis 2018;14:741-747.

10. Kothari SN, Borgert AJ, Kallies KJ, Baker MT, Grover BT. Long-term (>10-year) outcomes after laparoscopic Roux-en-Y gastric bypass.
Surg Obes Relat Dis 2017;13:972-978.

11. Tornese S, Aiolfi A, Bonitta G, et al. Remnant gastric cancer after Roux-en-Y gastric bypass: narrative review of the literature. Obes Surg 2019;29:2609-2613.

12. Mangan A, Le Roux CW, Miller NG, Docherty NG. Iron and vitamin D/calcium deficiency after gastric bypass: mechanisms involved and strategies to improve oral supplement disposition. Curr Drug Metab 2019;20:244-252.

13. Emous M, Wolffenbuttel BH, Totté E, van Beek AP. The short- to mid-term symptom prevalence of dumping syndrome after primary gastric-bypass surgery and its impact on health-related quality of life. Surg Obes Relat Dis 2017;13:1489-1500.

14. Geubbels N, Lijftogt N, Fiocco M, van Leersum NJ, Wouters MW, de Brauw LM. Meta-analysis of internal herniation after gastric bypass surgery. Br J Surg 2015;102:451-460.

15. Rheinwalt KP, Plamper A, Rückbeil MV, Kroh A, Neumann UP, Ulmer TF. One anastomosis gastric bypass-mini-gastric bypass (OAGBMGB) versus Roux-en-Y gastric bypass (RYGB): a mid-term cohort study with 612 patients. Obes Surg 2020;30:1230-1240.

16. Homan J, Betzel B, Aarts EO, et al. Vitamin and mineral deficiencies after biliopancreatic diversion and biliopancreatic diversion with duodenal switch: the rule rather than the exception. Obes Surg 2015;25: 1626-1632.

17. Huang CK, Goel R, Tai CM, Yen YC, Gohil VD, Chen XY. Novel metabolic surgery for type II diabetes mellitus: loop duodenojejunal bypass with sleeve gastrectomy. Surg Laparosc Endosc Percutan Tech 2013;23:481-485.

18. Brown WA, Ooi G, Higa K, Himpens J, Torres A; IFSO-appointed task force reviewing the literature on SADI-S/OADS. Single anastomosis duodenal-ileal bypass with sleeve gastrectomy/one anastomosis duodenal switch (SADI-S/OADS) IFSO position statement. Obes Surg 2018;28:1207-1216.

19. Dallegrave Marchesini JC. End-to-side duodeno-jejunostomy with half-and-half biliopancreatic limb for the treatment of type 2 diabetes: a proposal for a simpler technique. Obes Surg 2007;17:138-139.

20. Gebelli JP, Gordejuela AG, Ramos AC, et al. SADI-S with right gastric artery ligation: technical systematization and early results. Arq Bras Cir Dig 2016;29 Suppl 1(Suppl 1):85-90.

21. Kasama K, Tagaya N, Kanehira E, et al. Laparoscopic sleeve gastrectomy with duodenojejunal bypass: technique and preliminary results. Obes Surg 2009;19:1341-1345.

22. Maciejewski ML, Arterburn DE, Van Scoyoc L, et al. Bariatric surgery and long-term durability of weight loss. JAMA Surg 2016;151:10461055.

23. Nor Hanipah Z, Hsin MC, Liu CC, Huang CK. Laparoscopic loop duodenaljejunal bypass with sleeve gastrectomy in type 2 diabetic patients. Surg Obes Relat Dis 2019;15:696-702.

24. Sawaya RA, Jaffe J, Friedenberg L, Friedenberg FK. Vitamin, mineral, and drug absorption following bariatric surgery. Curr Drug Metab 2012;13:1345-1355. 
25. Fuqua BK, Vulpe CD, Anderson GJ. Intestinal iron absorption. J Trace Elem Med Biol 2012;26:115-119.

26. Bronner F. Mechanisms of intestinal calcium absorption. J Cell Biochem 2003;88:387-393.

27. Avgerinos KI, Spyrou N, Mantzoros CS, Dalamaga M. Obesity and cancer risk: emerging biological mechanisms and perspectives. Me- tabolism 2019;92:121-135.

28. Wojciechowska J, Krajewski W, Bolanowski M, Kręcicki T, Zatoński T. Diabetes and cancer: a review of current knowledge. Exp Clin Endocrinol Diabetes 2016;124:263-275.

29. Maestro $A$, Rigla $M$, Caixàs $A$. Does bariatric surgery reduce cancer risk?: a review of the literature. Endocrinol Nutr 2015;62:138-143. 